\title{
Upregulation of miR-501-5p activates the wnt/ $\beta$-catenin signaling pathway and enhances stem cell-like phenotype in gastric cancer
}

\author{
Dongmei Fan ${ }^{1 * \dagger}$, Baoqi Ren ${ }^{2 \dagger}$, Xiaojun Yang ${ }^{1}$, Jia Liu ${ }^{3}$ and Zhengzheng Zhang ${ }^{1}$
}

\begin{abstract}
Background: miRNAs are critical post-transcriptional regulators of gene expression and key mediators of tumourigenesis. miR-501-5p is newly identified to be involved in the tumor progression, but its biological role and mechanism remain largely unknown. This study is aimed to study the role of miR-501-5p in the progression of gastric cancer.

Methods: Real-time PCR analysis was used to determine miR-501-5p expression in gastric cancer cell lines, clinical tissues and 112 clinicopathologically characterized gastric cancer specimens. The role of miR-501-5p in maintaining gastric cancer stem cell like phenotype was examined by tumor-sphere formation assay and expression of stem cell markers. Luciferase reporter assay, cellular fractionation and western blot analysis were used to determined that miR-501-5p activated the wnt/ß-catenin signaling by directly targeting DKK1, NKD1 and GSK3 3 .

Results: Herein, our results revealed that miR-501-5p was markedly upregulated in gastric cancer cell lines and clinical tissues. High miR-501-5p levels predicted poor overall survival in gastric cancer patients. Gain-of-function and loss-of-function studies showed that ectopic expression of miR-501-5p enhanced the cancer stem cell-like phenotype in gastric cancer cells. Notably,wnt/ $\beta$-catenin signaling was hyperactivated in gastric cancer cells that overexpress miR-501-5p, and mediated miR-501-5p-induced cancer stem cell-like phenotype. Furthermore, miR-501-5p directly targeted and suppressed multiple repressors of the wnt/ $\beta$-catenin signaling cascade, including DKK1, NKD1 and GSK3 $\beta$. These results demonstrate that miR-501-5p maintains constitutively activated wnt/ $\beta$-catenin signaling by directly targeting DKK1, NKD1 and GSK3 3 , which promotes gastric cancer stem cell like phenotype.
\end{abstract}

Conclusions: Taken together, our findings reveal a new regulatory mechanism of miR-501-5p and suggest that miR501-5p might be a potential target in gastric cancer therapy.

Keywords: miR-501-5p, Gastric cancer, Wnt/ $\beta$-catenin signaling, Cancer stem cell

\section{Background}

Gastric cancer (GC) is the fifth most common malignancy and the third leading cause of cancer-related death worldwide. It is estimated that 951,600 new GC cases and 723,100 deaths occurred in 2012 [1]. In most countries, survival from stomach cancer remained in the narrow range of $25-30 \%$ [2]. Although therapeutic

\footnotetext{
* Correspondence: dongmeifan9@sina.com

${ }^{\dagger}$ Equal contributors

${ }^{1}$ Department of Gastroenterology, First Affiliated Hospital of Guangzhou University of Chinese Medicine, Guangzhou 510405, China

Full list of author information is available at the end of the article
}

methods are improving in surgical combined with radiotherapy and chemotherapy, the prognosis for advanced stage patients is still very poor [3, 4]. Recent studies have revealed that gastric cancer stem cells constitute a reservoir of selfsustaining cells with the exclusive ability to self-renew and cause tumor outgrowth [5]: Integral to the CSC model is a subpopulation at the apex of the hierarchy (usually comprising $<5 \%$ of the cancer), responsible for the formation, maintenance, and continued growth of the tumour [6]. However, the molecular mechanisms for the maintenance of GC stem cell phenotype remains largely unknown. 
The Wnt/ $\beta$-catenin signaling pathway is considered as a key player in the regulation of tissue homeostasis, organ size, tumorigenesis [7-9]. Upon activation, the wnt signals will stabilize and finally lead to accumulation of $\beta$-catenin. Activated $\beta$-catenin dissociates with $\mathrm{E}$ cadherin, dissembling the adherens and enters the nucleus to turn on the expression of target genes, most of which show invasion promotion functions [10]. Notably, recent advances have shown that the wnt/ $\beta$-catenin signaling pathway is involved in the maintenance of the GC stem cell population $[11,12]$. It has been reported that the pathway blocked by dickkopf WNT signaling pathway inhibitor 1 (DKK-1), naked cuticle homolog 1 (NKD1) and glycogen synthase kinase 3 beta (GSK3 $\beta$ ) caused a robust reduction in the activity of wnt/ $\beta$-catenin signaling and self-renewing capacity of gastric cancer cells [12-16]. Accordingly, the wnt/ $\beta$-catenin signaling pathway is considered as an important regulator in the maintenance of CSC population, and better understanding of the mechanisms that regulate wnt/ $\beta$-catenin pathway may provide new clues for more effective GC therapy.

MicroRNAs (miRNAs) are a class of highly conserved, small noncoding RNAs that are approximately 22 nucleotides in length. Typically, miRNAs bind to the 3 '-untranslated regions (3'-UTRs) of mRNAs, guide the formation of miRNA-mRNA-induced silencing complexes and lead to the degradation or inhibit the translation of the targeted mRNAs [17, 18]. Bioinformatic predictions indicate that miRNAs regulate more than $30 \%$ of the protein-coding genes [19]. The dysregulation of miRNA expression appears to be a general trait of GC [20]. Thus, it is of particular interest to identify miRNAs that might interfere with wnt/ $\beta$-catenin signaling and thereby lead to the self-renewal of GC stem cells.

In the present study, we sought to elucidate the the effect of miR-501-5p on wnt/ $\beta$-catenin signaling in GC, as well as the related molecular mechanism by which miR-501-5p affected wnt/ $\beta$-catenin signaling. Our data showed that miR-501-5p was upregulated in GC tissues and correlated with a more aggressive phenotype of GC in patients. miR-501-5p activates the wnt/ $\beta$-catenin pathway by directly targeting DKK1, NKD1 and GSK3 $\beta$, and consequently enhances stem cell-like phenotype of GC.

\section{Methods}

\section{Tissues and cells}

Fresh GC tissue samples from GC patients, and their matched adjacent non-tumor gastric mucosal tissues ( $>5 \mathrm{~cm}$ laterally from the edge of tumor region) were obtained from the First Affiliated Hospital of Guangzhou University of Chinese Medicine. The samples had been clinically and histopathologically diagnosed according to the World Health Organization criteria. Tumor and non-cancerous tissues were confirmed histologically by hematoxylin and eosin staining. All samples were collected from consenting individuals according to the protocols approved by the Ethics Review Board at Guangzhou University of Chinese Medicine. GC cell lines SGC-7901, HGC-27, MGC-803, MKN-28 and BGC-823 were routinely maintained in DMEM medium (Invitrogen, Carlsbad, CA) supplemented with 10\% fetal bovine serum (HyClone, Logan, UT).

\section{Plasmids and oligonucleotides}

The TOP/FOP Luciferase Reporting system was purchased from lifeome (Oceanside, CA, USA). miR-501-5p and miR-501-5p antagonist (antagomiR-501-5p, RiboBio, Guangzhou, China) were used to overexpress or inhibit miR-501-5p. The 3'UTRs of human DKK1, NKD1 and GSK3 $\beta$ were generated by PCR amplification and subcloned into the pGL3 vector plasmid (Promega, Madison, WI). The primers are as the following:

\section{DKK1-3' UTR-luc-up, 5' -GCCCCGCGGCACTAAA CCAGCTATCCA-3' ; \\ DKK1-3' UTR-luc-dn, 5' -GCCCTGCAGTAGGCAG TGCAGCACCTT-3' ; \\ NKD1-3' UTR-luc-up, 5' -GCCCCGCGGGAGCCTG GAGAAACCTGAAA-3' ; \\ NKD1-3' UTR-luc-dn, 5' -GCCCTGCAGCCCACAT CAACAAGCTCCCT-3' ; \\ GSK3B-3' UTR-luc-up, 5' -GCCCCGCGGTGCCTC AAAGTAGTCCAT-3' ; \\ GSK3B-3' UTR-luc-dn, 5' -GCCCTGCAGGTGTTT GGCTCTGTGATT-3' ;}

The siRNA sequences were as followings:

ß-catenin-siRNA: $5^{\prime}$-CCAUUGUUUGUGCAGCUGC UU-3' ;

DKK1-siRNAi: 5' -ACACUUGUCAGAGACACUAAA-3' ; NKD1-siRNAi: 5' -CCACUUAAACAAGCGUGGUUU-3' ; GSK3B-siRNAi: 5' -CCACUCAAGAACUGUCAAGUA-3' .

\section{Western blot analysis}

Cells were harvested in cell lysis buffer (Cell Signaling Technology; Cat\#: 9803) and heated for $5 \mathrm{~min}$ at $100{ }^{\circ} \mathrm{C}$. Equal quantities of denatured protein samples were resolved on $10 \%$ SDS-polyacrylamide gels and then transferred onto polyvinylidene difluoride membranes (Roche). After blocking with 5\% non-fat dry milk in Tris-buffered saline/0.05\% Tween 20 (TBST), the membrane was incubated with a specific primary antibody followed by a horseradish peroxidase-conjugated secondary antibody. Proteins were visualized using ECL reagents (Pierce). The antibodies used were as follows: anti- $\beta$-catenin, anti-DKK1, anti-NKD1 and anti-GSK3 $\beta$ (Abcam, Cambridge, MA, USA), and p-GSK3 $\beta$ (Ser9) (Cell Signaling Technology). 
The membranes were stripped and reprobed with an anti- $\alpha$-tubulin antibody (Sigma-Aldrich, St. Louis, MO, USA) as the loading control.

\section{MiRNA extraction and real-time quantitative PCR}

Total miRNA from cultured cells and fresh surgical gastric cancer tissues was extracted using a mirVana miRNA Isolation Kit (Ambion, Austin, TX, USA) according to the manufacturer's instructions. We synthesised cDNA from $10 \mathrm{ng}$ total RNA using a TaqMan miRNA reverse transcription kit (Applied Biosystems, Foster City, CA, USA), and quantified the expression levels of miR-501-5p using a miRNA-specific TaqMan MiRNA Assay Kit (Applied Biosystems). miRNA expression was defined based on the threshold cycle $(\mathrm{Ct})$, and relative expression levels were calculated as $2^{-[(\mathrm{Ct} \text { of miR- }}$ 501-5p) - (Ct of U6)] after normalization with reference to expression of U6 small nuclear RNA.

\section{Sphere formation assays}

$1 \times 10^{3}$ cells were seeded in 6-well ultra low cluster plates (Corning, NY) and about 10 cells were seeded in 24-well ultra low cluster plates (Corning, NY) for 15 days. Spheres were cultured in DMEM/F12 serumfree medium (Invitrogen, Grand Island, NY) supplemented with 2\% B27 (Invitrogen, Grand Island, NY), $20 \mathrm{ng} / \mathrm{ml}$ of EGF, and $20 \mathrm{ng} / \mathrm{ml}$ of bFGF (PeproTech, Offenbach, Germany), $0.4 \%$ bovine serum albumin (BSA) (Sigma, St. Louis, MO, USA), and $5 \mu \mathrm{g} / \mathrm{ml}$ insulin.

\section{Luciferase assays}

Cells $\left(4 \times 10^{4}\right)$ were seeded in triplicate in 24 -well plates and cultured for $24 \mathrm{~h}$. Cells were transfected with $100 \mathrm{ng}$ TOP/FOP reporter luciferase plasmid, or pGL3DKK1-3'UTR, pGL3-NKD1-3'UTRor pGL3-GSK3B-3' UTR luciferase plasmids, plus 5 ng pRL-TK Renilla plasmid (Promega) using Lipofectamine 2000 (Invitrogen) according to the manufacturer's recommendation.
Luciferase and Renilla signals were measured $36 \mathrm{~h}$ after transfection using a Dual Luciferase Reporter Assay Kit (Promega) according to the manufacturer's protocol.

\section{Statistical analyses}

All statistical analyses were carried out using SPSS statistical software (SPSS Inc., Chicago, IL, USA). The 2-tailed Student's t-test was used to evaluate the significance of the differences between two groups of data in all pertinent experiments; a $P$ value $<0.05$ was considered significant.

\section{Results}

miR-501-5p is upregulated in human gastric cancer tissues and cell lines

By analysis of the The Cancer Genome Atlas (TCGA) Stomach adenocarcinoma miRNA sequencing data sets, we found that miR-501-5p levels were significantly upregulated in human gastric cancer tissues $(n=395)$ compared with that in normal gastric tissues $(n=41)$ $(p<0.001)$ (Fig. 1a). We further verified this result in gastric cancer cell lines and paired tissues by realtime PCR analysis. As shown in Fig. $1 \mathrm{~b}$ and c, miR501-5p levels were differentially increased in 5 gastric cancer cell lines than that in normal gastric epithelial cell (NGEC), and in 10 gastric cancer tissues (T) compared to that in the paired adjacent normal tissues (ANT). Collectively, these results suggest that miR-501-5p is upregulated and might be involved in human gastric cancer progression.

\section{High miR-501-5p predicts poor prognosis}

We further assessed the clinical significance of miR-501$5 p$ expression levels in 112 gastric cancer tissues. As shown in Fig. 2a, miR-501-5p expression was markedly increased in 112 gastric cancer samples compared with that in 12 non-cancerous gastric tissues. Importantly, patients with high miR-501-5p expression had a significant poor overall survival compared to patients with low
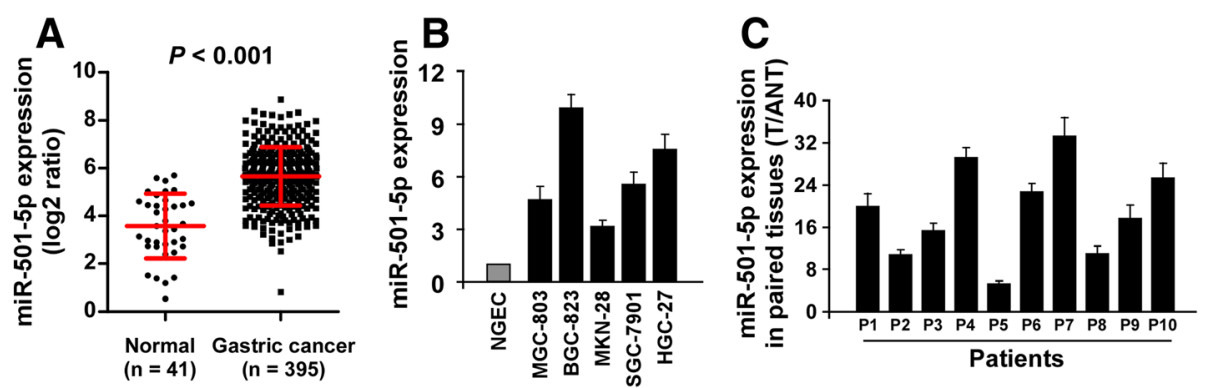

Fig. 1 miR-501-5p is upregulated in human gastric cancer tissues and cell lines. a miR-501-5p levels remained low in normal gastric tissues but became dramatically elevated in gastric cancer tissues by analyzing The Cancer Genome Atlas (TCGA) gastric cancer miRNA sequencing data sets (Normal, $n=41$; gastric cancer, $n=395$ ). $P<0.001$, 2-tailed Student's $t$-test. $\mathbf{b}$ and $\mathbf{c}$ Real-time PCR analysis of miR-501-5p expression in normal gastric epithelial cell (NGEC) and 5 cultured gastric cancer cell lines (b), and in 10 pairs of gastric cancer samples (T) and adjacent normal tissues (ANT) (c). Transcript levels were normalized by U6 expression. Error bars represent the mean \pm s.d. of three independent experiments. ${ }^{*} P<0.05$ 

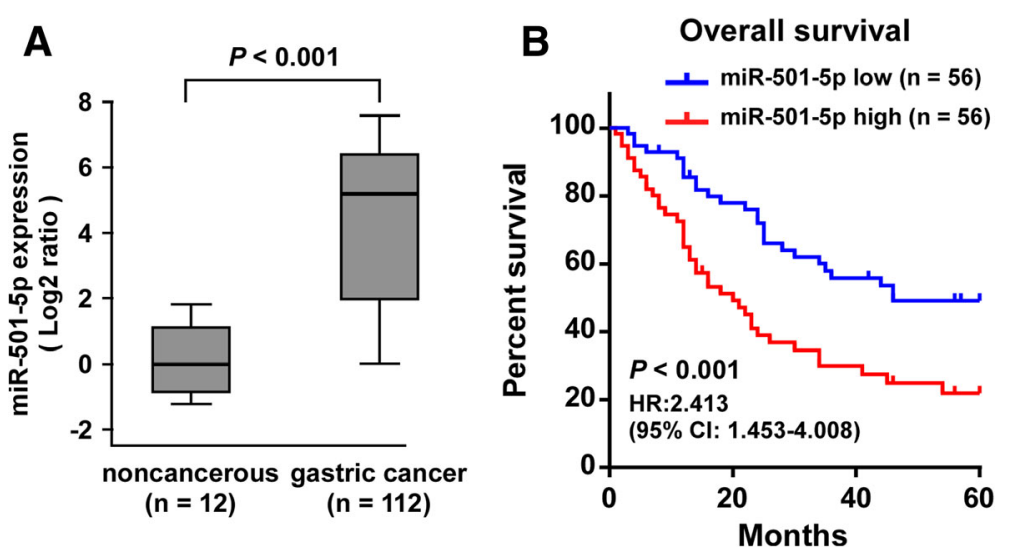

Fig. 2 High miR-501-5p predicts poor prognosis. a Real-time PCR analysis of miR-501-5p expression in 112 freshly collected gastric cancer tissues compared to that in 12 non-cancerous gastric tissues. Transcript levels were normalized to U6 expression. Boundaries of boxes represent lower and upper quartiles, respectively. Lines within boxes and whiskers denote median and extremum, respectively. $P<0.001,2$-tailed Student's $t$-test. b Kaplan-Meier analysis of 5-year overall survival curves of patients with gastric cancer with high miR-501-5p expression ( $>$ median, $n=56)$ versus low miR-501-5p expression ( $<$ median, $n=56$ ) [hazard ratio $(H R)=2.413 ; 95 \%$ confidence interval $(C \mathrm{Cl}): 1.453-4.008]$. $P<0.001$, log-rank test

miR-501-5p expression $(P<0.001$; hazard ratio $=2.413$, $95 \% \mathrm{CI}=1.453-4.008$; Fig. 2b).

\section{miR-501-5p enhances the stem cell-like phenotype in gastric cancer cells}

It has been noted that aquisition of stem cell-like phenotype of gastric cancer cells is crucial for the malignance and high frequency of relapse [5]. We then investigated the role of miR-501-5p upregulation in the self-renewal ability of gastric cancer cells. Gastric cancer cell lines MGC-803 and SGC-7901 were engineered to overexpress or silence miR-501-5p by transfection of miR-501-5p mimic or antagomiR-5015p (Additional file 1: Figure S1). Notably, overexpression of miR-501-5p robustly promoted gastric cancer cells cultured in suspension to generate approximately two-fold more tumor spheres and higher cell content, compared with the spheres formed by control cells until day 15 (Fig. 3a-c). Conversely, miR-501-5p-silenced cells formed $\sim 3$-fold fewer spheres and lower cell content, compared with control cells (Fig. 3a-c). Moreover, flow cytometry assays revealed a higher percentage of the side-population of cells in the miR501-5p overexpressing cells but a lower percentage in the miR-501-5p-inhibited cells compared to control (Additional file 2: Figure S2). In addition, overexpression of miR-501-5p increased, while downregulation of miR-501-5p reduced the expression levels of different stem cell regulators including CD44, CD133, Bmi1, Nanog, MYC and SOX2 (Fig. 3d). Thus, our results indicate that miR-501-5p promotes the stem cell-like phenotype in gastric cancer.
miR-501-5p activates wnt/ $\beta$-catenin signaling pathway Since wnt $/ \beta$-catenin signaling is one of the most important pathways in maintaining stem cell phenotype and frequently activated in gastric cancer, we then examined the role of miR-501-5p in wnt/ $\beta$-catenin signaling pathway. As shown in Fig. 4a, we found that miR-501-5p overexpression significantly increased, but silencing of miR-501-5p reduced the TOP/FOP luciferase reporter activity. In addition, cellular fractionation and western blot analysis revealed that overexpression of miR-501$5 p$ increased nuclear accumulation of $\beta$-catenin, while silencing of miR-501-5p reduced nuclear $\beta$-catenin expression (Fig. 4b). Moreover, real-time PCR analysis revealed that miR-501-5p upregulated, but downregulation of miR-501-5p repressed the expression levels of the multiple wnt $/ \beta$-catenin downstream genes (Fig. 4c). Collectively, our results suggest that miR$501-5 \mathrm{p}$ activates $\mathrm{Wnt} / \beta$-catenin signaling pathway in gastric cancer.

We further investigated the functional significance of wnt $/ \beta$-catenin signaling activation in miR-501-5p-mediated self-renewal of gastric cancer cells by silencing $\beta$ catenin or expressing TCF4-dn in miR-501-5p-overexpressing MGC-803 and SGC-7901 cells. As expected, the stimulatory effect of miR-501-5p on TOP/FOP luciferase reporter activity was impaired by silencing $\beta$ catenin or expressing TCF4-dn (Fig. 4d). Moreover, sphere formation assays indicated that silencing $\beta$ catenin or expressing TCF4-dn abrogated the promotive effects of miR-501-5p on self-renewal of gastric cancer cells (Fig. 4e). Thus, these results reveal that activation of wnt $/ \beta$-catenin signaling is essential for miR-501-5ppromoted stem cell like phenotype in gastric cancer. 

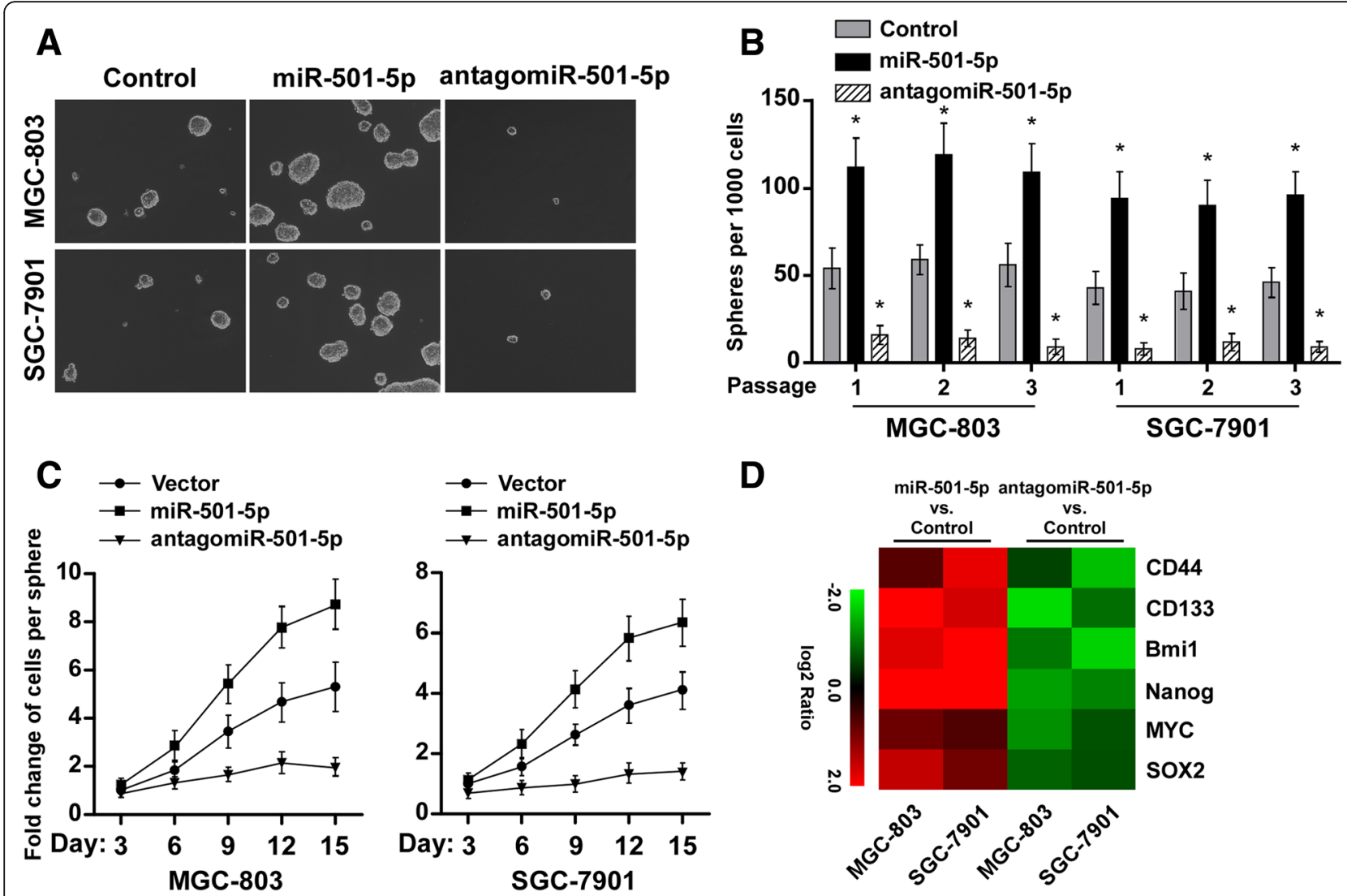

D

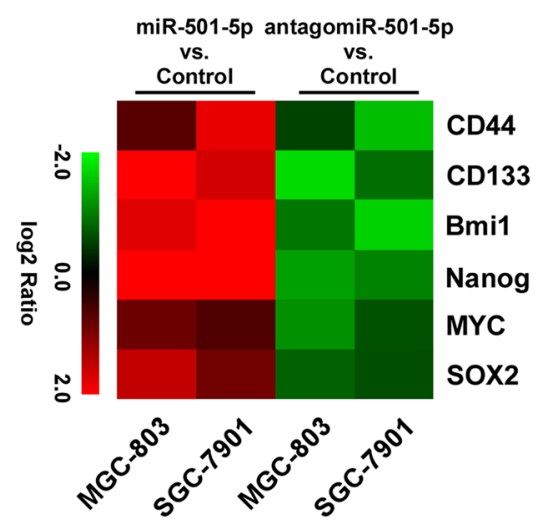

Fig. 3 miR-501-5p enhances the stem cell-like phenotype in gastric cancer cells. a Representative micrographs of tumor spheres formed by indicated cells. $\mathbf{b}$ Histograms showing the mean number of spheres formed by the indicated cells from different passages. $\mathbf{c}$ The fold change in the number of cells per sphere on the indicated days. $\mathbf{d}$ Real-time PCR analysis revealed that miR-501-5p regulates the expression levels of numerous stem cell regulators. The pseudocolors represent the intensity scale of expression in miR-501-5p vs. control-transfected cells or antagomiR-501-5p vs. controltransfected cells generated by $\log _{2}$ transformation. Error bars represent the mean \pm s.d. of three independent experiments. ${ }^{*} P<0.05$

miR-501-5p directly targets multiple repressors of wnt/ $\beta$ catenin signaling pathway

Interestingly, using the publicly available algorithms TargetScan and miRanda, we found that multiple repressors of wnt/ $\beta$-catenin signaling, i.e., DKK1, NKD1 and GSK3B might be potential targets of miR-501-5p (Fig. 5a). Western blot analysis revealed that overexpression of miR-501-5p markedly reduced the expression levels of DKK1, NKD1 and GSK3 3 . In contrast, miR501-5p inhibition increased them, suggesting that miR501-5p negatively regulated these proteins (Fig. 5b). Meanwhile, overexpression of miR-501-5p increased, but inhibition of miR-501-5p repressed $\beta$-catenin expression in gastric cancer cells (Fig. 5b). Luciferase assay showed that miR-501-5p overexpression attenuated, while inhibition of miR-501-5p elevated the reporter activities driven by the 3'UTRs of DKK1, NKD1 and GSK3B transcripts (Fig. 5c). However, ectopic expression of the mutant miR-501-5p did not exhibit repressive effects on the reporter activities driven by the 3'UTRs of these transcripts (Fig. 5c). Moreover, microribonucleoprotein
(miRNP) immunoprecipitation (IP) assay revealed a selective association of miR-501-5p with DKK1, NKD1 and GSK3B, but not with GAPDH (Fig. 5d), further indicating the specific effects of miR-501-5p on these targets. In addition, individual silencing of these targets potently rescued the TOP/FOP luciferase reporter activity and self-renewal ability in miR-501-5p-inhibited cells (Fig. 5e and f), demonstrating that DKK1, NKD1 and GSK3 $\beta$ were functional effectors of miR-501-5p on regulating wnt $/ \beta$-catenin signaling activation and stem celllike phenotype in gastric cancer. Collectively, our results suggest that miR-501-5p activates wnt/ $\beta$-catenin signaling to enhance stem cell-like phenotype in gastric cancer by directly targeting DKK1, NKD1 and GSK3 $\beta$.

\section{Discussion}

MicroRNAs have been demonstrated to negatively regulate target mRNAs in a sequence-specific manner, and are key regulators in a wide variety of oncogenic processes, such as cell proliferation, differentiation, invasion and metastasis, functioning as either tumor suppressors 


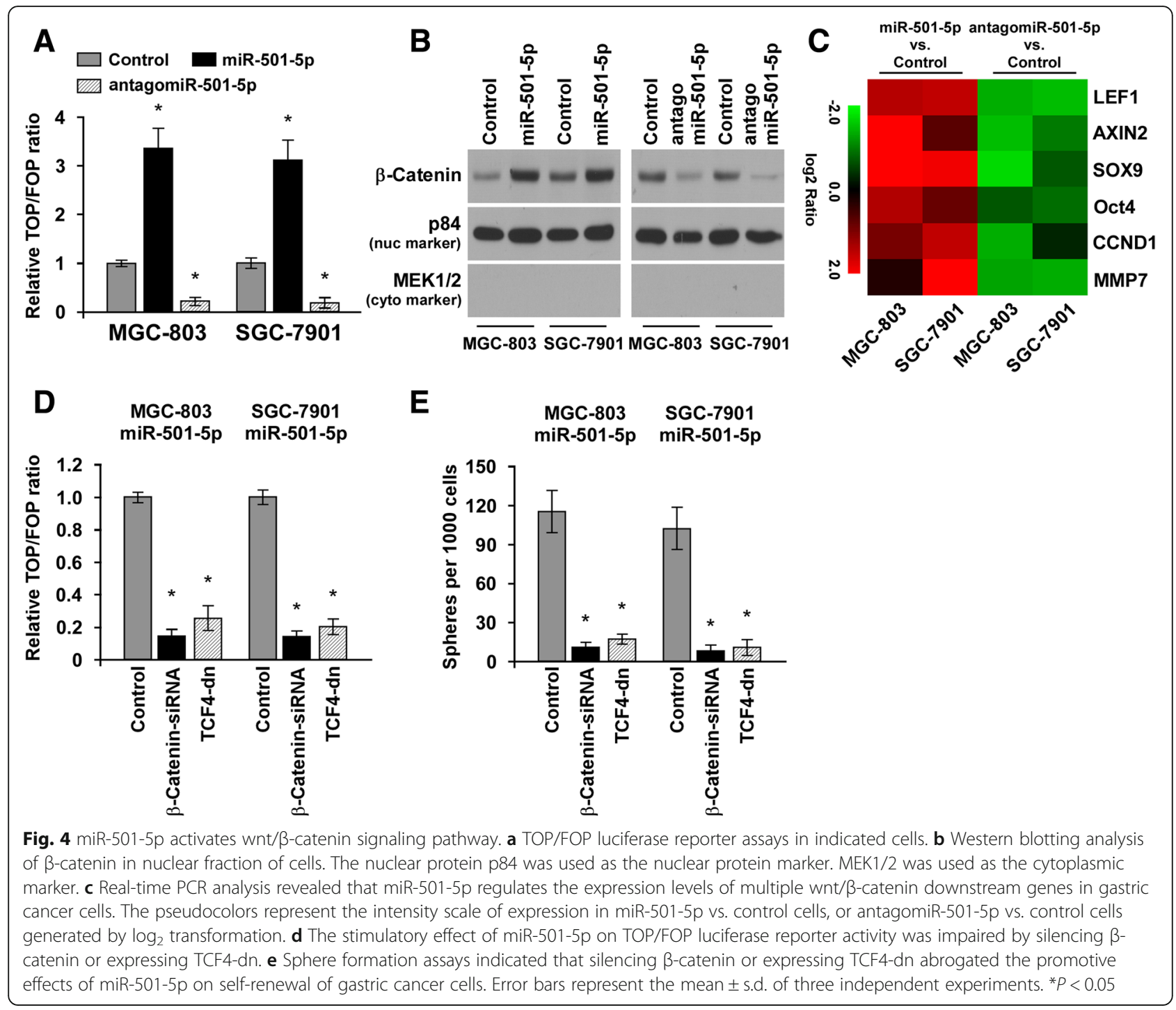

or oncogenes [21-23]. Therefore, elucidating the underlying mechanism of miRNAs in tumor development may provide valuable diagnostic and therapeutic strategies for malignancy. Previous evidence has demonstrated that miR-501-5p is overexpressed in human hepatocellular carcinoma, and upregulation of miR-501-5p contributes to cancer cell proliferation, migration, invasion and drug resistance via different mechanisms [24]. Herein, combining with the TCGA gastric cancer microRNA data set analysis, our results revealed that miR-501-5p was markedly upregulated in GC tissues compared with paired adjacent normal tissues, and was significantly correlated with a more aggressive phenotype of GC in patients.

Gastric cancer continues to be a highly lethal malignancy, despite the use of multimodal treatment approaches. The CSC model has been proposed to explain the high rate of relapse and subsequent resistance of cancer to current systemic treatments [25]. CSCs have been identified in many solid malignancies, including gastric cancer, and have significant clinical implications, as targeting the CSC population may be essential in preventing the recurrence and spread of a tumour [26, 27]. Herein, we found that miR-501-5p acted as a potent CSC-promoting factor in GC. Overexpression of miR501-5p promoted the self-renewal of GC cells and upregulated the pluripotency associated markers, including CD133, Bmi1, Nanog, MYC and Sox2. More importantly, inhibition of miR-501-5p by antagomiR-501-5p potentially reduced self-renewal of GC cells. Thus, our results suggest that upregulation of miR-501-5p in GC is involved in the malignant progression of GC, and propose that miR-501$5 \mathrm{p}$ might be a potential therapeutic target for human gastric cancer.

Accumulating evidence revealed that cancer stem cells have higher metastatic potency by inducing Epithelialmesenchymal transition (EMT) [28]. Notably, Yoshida 


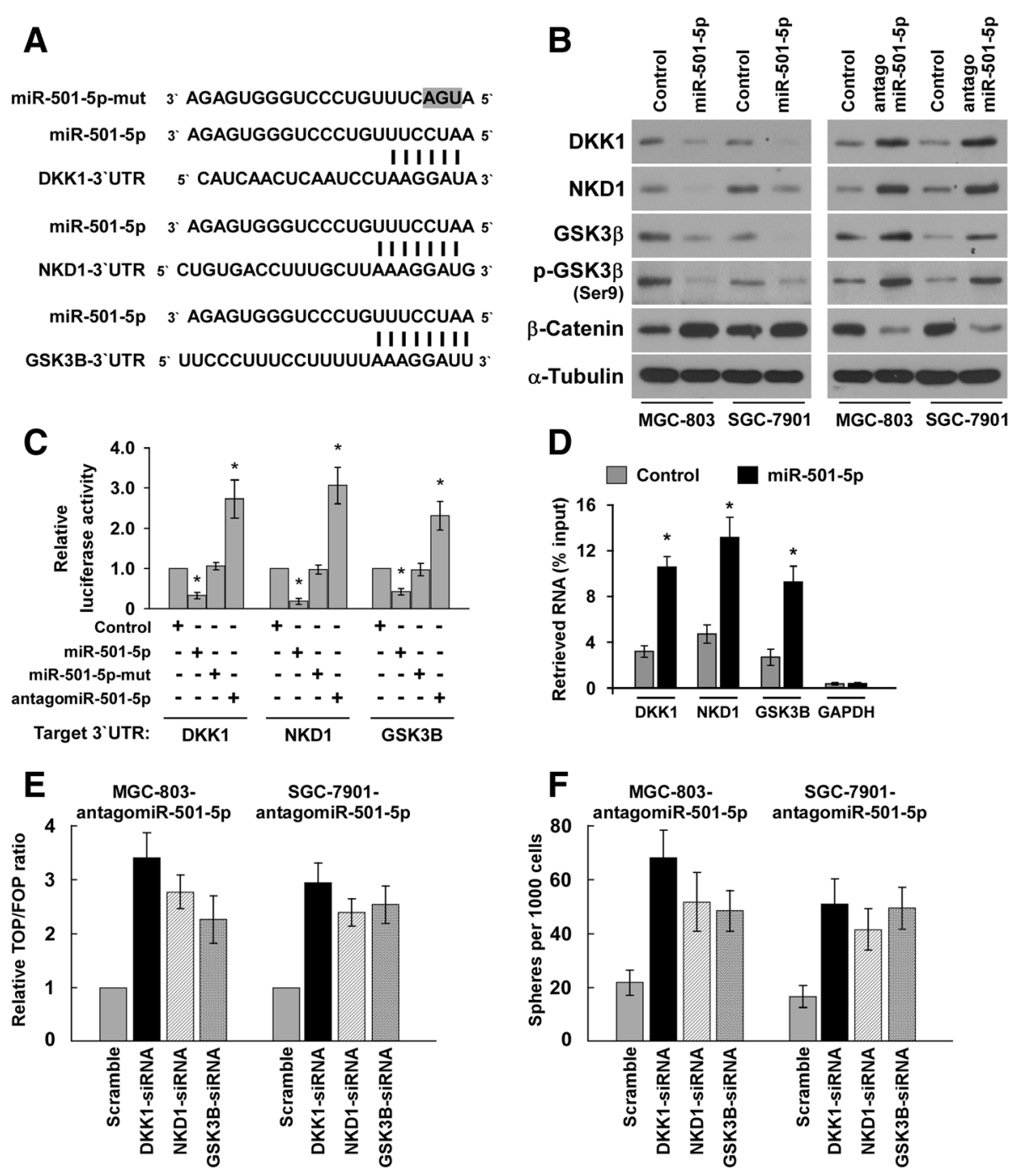

Fig. 5 miR-501-5p directly targets multiple repressors of wnt/ $\beta$-catenin signaling pathway. a Predicted miR-501-5p target sequence in $3^{\prime} U$ TRs of DKK1, NKD1 and GSK3B. The mutated miR-501-5p containing three altered nucleotides were indicated. $\mathbf{b}$ Western blots of DKK1, NKD1, GSK3 $\beta, p-G S K 3 \beta$ (Ser9) and $\beta$-catenin expression. a-Tubulin served as the loading control. c Luciferase assay of pGL3-DKK1-3'UTR, pGL3-NKD1-3'UTR or pGL3-GSK3B3'UTR reporter in the miR-501-5p-, antagomiR-501-5p-, mutant miR-501-5p- and control-transfected MGC-803 cells. d MiRNP IP assay showing the association between miR-501-5p and DKK1, NKD1 and GSK3B transcripts in MGC-803 cells. GAPDH served as the negative control. e Individual silencing of DKK1, NKD1 and GSK3B potently rescued the TOP/FOP luciferase reporter activity and self-renewal ability in miR-501-5p-inhibited gastric cancer cells (Fig. $5 \mathbf{e}$ and $\mathbf{f}$ ), demonstrating that these genes were functional effectors of miR-501-5p on regulating wnt/ $\beta$-catenin signaling activation and stem celllike phenotype in gastric cancer. Error bars represent the mean \pm s.d. of three independent experiments. ${ }^{*} P<0.05$

et al. recently found that oxidative stress-induced canonical Wnt activation play an important role in the heterogeneous cancer stem cell population at the invasive area by regulating CD44 and c-Myc [29]. Herein, we found that miR-501-5p increased the stemness in gastric cancer by activating the Wnt/ $\beta$-catenin signaling and increasing the expression of CD44 and c-Myc. Thus, miR501-5p might contribute to gastric cancer metastasis, and this hypothesis remains to be examined. For example, does miR-501-5p induce EMT; is miR-501-5p increased at the invasive edge of gastric cancer tissues; does miR-501-5p regulate the heterogeneous cancer stem cell population; these questions remain to be answer. Moreover, it was reported that Wnt activation could be induced by micro-environmental stress [29]. Therefore, it would be interesting to investigate whether micro-environmental stress is responsible for miR-501$5 \mathrm{p}$ upregulation in gastric cancer.

Several signaling pathways, including the wnt/ $\beta$-catenin, Notch, and PI3K/Akt pathways, have been found to be aberrantly activated and play vital roles in the development and progression of gastric cancer [11, 30, 31] 
Among these pathways, the functions of wnt $/ \beta$-catenin signaling in gastric cancer development and progression have been well documented. TCF4 and coactivator $\beta$ catenin are two key downstream effectors of the wnt $/ \beta$ catenin signaling pathway, and considered as potent oncogenes in tumor progression [7]. Notably, unlike in other cancer types, the mutations in $\beta$-catenin are rare, but the expression and/or nuclear localization of $\beta$-catenin is often abnormal in gastric cancer, indicating a constitutive activation of $\mathrm{Wnt} / \beta$-catenin signaling $[7,11,12]$. However, how cancer cells evade the negative regulation by $\mathrm{wnt} / \beta$-catenin signaling cascade, leading to constitutively activated $\beta$-catenin/TCF remains unclear. Herein, we demonstrated that miR-501-5p was substantially overexpressed in gastric cancer and induced hyper-activation of $\beta$-catenin/TCF via directly targeting DKK1, NKD1 and GSK3 $\beta$. Therefore, our findings not only suggest activation of $\mathrm{Wnt} / \beta$-catenin pathway contributes to the malignant behavior of gastric cancer, but also reveal a novel mechanism for activation of wnt/ $\beta$-catenin pathway involving miR-501-5p in gastric cancer.

It was reported that GSK-3 $\beta$ exhibited both oncogenic and tumor-suppressive roles in the context of different cancer types. For example, Tang et al. found that loss of GSK-3 $\beta$ expression activated $\beta$-Catenin to upregulate the expression of miR-183-96-182 cluster, leading to proliferation and migration of gastric cancer [32]. In contrast, inhibition of GSK3 $\beta$ compromises the survival, proliferation and invasion of glioblastoma cells [33]. Herein, we found that miR-501-5p repressed GSK3 $\beta$ to activate $\mathrm{Wnt} / \beta-\mathrm{Ca}$ tenin pathway and enhance gastric cancer stem cell phenotype, further suggesting a tumor-repressive role of GSK3 $\beta$ in gastric cancer. Moreover, the role of miR-501$5 \mathrm{p}$ in glioblastoma remains to be further examined in future. Appropriate reference and discussion have been incorporated into revised manuscript.

\section{Conclusions}

In conclusion, our study has revealed that miR-501-5p upregulation plays an important role in gastric cancer progression and miR-501-5p is a critical activator of Wnt/ $\beta$-catenin signaling by targeting DKK1, NKD1 and GSK3 $\beta$. Understanding the precise role of miR-501-5p in gastric cancer pathogenesis and in the $w n t / \beta$-catenin signaling pathway promises to increase our knowledge of the biological basis of cancer development and may also facilitate the development of new therapeutic strategies against gastric cancer.

\section{Additional files}

Additional file 1: Figure S1. Real-time PCR analysis of miR-501-5p in miR-501-5p-overexpressing, miR-501-5p-silenced and control MGC-803 and
SGC-7901 cell lines. Transcript levels were normalized to U6 expression. (TIF $97 \mathrm{~kb}$ )

Additional file 2: Figure S2. Hoechst 33342 dye exclusion assay showing that overexpressing miR-501-5p promoted, whereas inhibition of miR-501-5p attenuated, the side-population cells in gastric cancer cells. (TIF $193 \mathrm{~kb}$ )

\section{Authors' contributions}

DF and BR carried out most of the experimental work. DF and XY examined miR-501-5p expression in cell lines and clinical samples. BR and $J$ conducted luciferase reporter assay and western blot analysis. BR and ZZ conducted Real-time PCR analysis and data analysis. DF supervised the project and wrote the manuscript. All authors read and approved the final manuscript.

\section{Competing interests}

The authors declare that they have no competing interests.

\section{Author details}

'Department of Gastroenterology, First Affiliated Hospital of Guangzhou University of Chinese Medicine, Guangzhou 510405, China. ${ }^{2}$ Medical Department of Guangdong Hospital of Traditional Chinese Medicine, Guangzhou 510405, China. ${ }^{3}$ Third Affiliated Hospital of Guangzhou Medical College, Guangzhou, China.

Received: 23 December 2015 Accepted: 11 April 2016

Published online: 15 November 2016

References

1. Torre LA, Bray F, Siegel RL, Ferlay J, Lortet-Tieulent J, Jemal A. Global cancer statistics, 2012. CA Cancer J Clin. 2015;65(2):87-108.

2. Allemani C, Weir HK, Carreira H, Harewood R, Spika D, Wang XS, Bannon F, Ahn JV, Johnson CJ, Bonaventure A, et al. Global surveillance of cancer survival 1995-2009: analysis of individual data for 25,676,887 patients from 279 population-based registries in 67 countries (CONCORD-2). Lancet. 2015; 385(9972):977-1010.

3. Cui J, Yin Y, Ma Q, Wang G, Olman V, Zhang Y, Chou WC, Hong CS, Zhang C, Cao S, et al. Comprehensive characterization of the genomic alterations in human gastric cancer. Int J Cancer. 2015;137(1):86-95.

4. Oliveira C, Pinheiro H, Figueiredo J, Seruca R, Carneiro F. Familial gastric cancer: genetic susceptibility, pathology, and implications for management. Lancet Oncol. 2015;16(2):e60-70.

5. Brungs D, Aghmesheh M, Vine KL, Becker TM, Carolan MG, Ranson M. Gastric cancer stem cells: evidence, potential markers, and clinical implications. J Gastroenterol. 2016;51(4):313-26.

6. Jang BG, Lee BL, Kim WH. Prognostic significance of leucine-rich-repeatcontaining G-protein-coupled receptor 5, an intestinal stem cell marker, in gastric carcinomas. Gastric Cancer. 2016;19(3):767-77.

7. Chiurillo MA. Role of the Wnt/beta-catenin pathway in gastric cancer: an indepth literature review. World J Exp Med. 2015;5(2):84-102.

8. Clevers H, Loh KM, Nusse R. Stem cell signaling. An integral program for tissue renewal and regeneration: Wnt signaling and stem cell control. Science. 2014;346(6205):1248012.

9. Clevers H, Nusse R. Wnt/beta-catenin signaling and disease. Cell. 2012; 149(6):1192-205.

10. Tian X, Liu Z, Niu B, Zhang J, Tan TK, Lee SR, Zhao Y, Harris DC, Zheng G. Ecadherin/beta-catenin complex and the epithelial barrier. J Biomed Biotechnol. 2011;2011:567305.

11. Mao J, Fan S, Ma W, Fan P, Wang B, Zhang J, Wang H, Tang B, Zhang Q, Yu $X$, et al. Roles of Wnt/beta-catenin signaling in the gastric cancer stem cells proliferation and salinomycin treatment. Cell Death Dis. 2014;5:e1039.

12. Cai $C$, Zhu $X$. The Wnt/beta-catenin pathway regulates self-renewal of cancer stem-like cells in human gastric cancer. Mol Med Rep. 2012;5(5):1191-6.

13. Katoh M. Molecular cloning, gene structure, and expression analyses of NKD1 and NKD2. Int J Oncol. 2001;19(5):963-9.

14. Guo J, Cagatay T, Zhou G, Chan CC, Blythe S, Suyama K, Zheng L, Pan K, Qian C, Hamelin R, et al. Mutations in the human naked cuticle homolog NKD1 found in colorectal cancer alter Wnt/Dvl/beta-catenin signaling. PLoS One. 2009;4(11):e7982.

15. Niehrs C. Head in the WNT: the molecular nature of Spemann's head organizer. Trends Genet. 1999;15(8):314-9. 
16. Dajani R, Fraser E, Roe SM, Yeo M, Good VM, Thompson V, Dale TC, Pearl LH. Structural basis for recruitment of glycogen synthase kinase 3 beta to the axin-APC scaffold complex. EMBO J. 2003;22(3):494-501.

17. Leung AK. The whereabouts of microRNA actions: cytoplasm and beyond. Trends Cell Biol. 2015;25(10):601-10.

18. Su Z, Yang Z, Xu Y, Chen Y, Yu Q. MicroRNAs in apoptosis, autophagy and necroptosis. Oncotarget. 2015;6(11):8474-90.

19. Oulas A, Karathanasis N, Louloupi A, Pavlopoulos GA, Poirazi P, Kalantidis K, Iliopoulos I. Prediction of miRNA targets. Methods Mol Biol. 2015;1269:207-29.

20. Guo LL, Song CH, Wang P, Dai LP, Zhang JY, Wang KJ. Competing endogenous RNA networks and gastric cancer. World J Gastroenterol. 2015; 21(41):11680-7.

21. Bartel DP. MicroRNAs: genomics, biogenesis, mechanism, and function. Cell. 2004;116(2):281-97.

22. Hata A, Kashima R. Dysregulation of microRNA biogenesis machinery in cancer. Crit Rev Biochem Mol Biol. 2016;51(3):121-34.

23. Esquela-Kerscher A, Slack FJ. Oncomirs - microRNAs with a role in cancer. Nat Rev Cancer. 2006;6(4):259-69.

24. Huang DH, Wang GY, Zhang JW, Li Y, Zeng XC, Jiang N. MiR-501-5p regulates CYLD expression and promotes cell proliferation in human hepatocellular carcinoma. Jpn J Clin Oncol. 2015;45(8):738-44.

25. Vermeulen L, de Sousa e Melo F, Richel DJ, Medema JP. The developing cancer stem-cell model: clinical challenges and opportunities. Lancet Oncol. 2012:13(2):e83-9.

26. Dewi DL, Ishii H, Kano Y, Nishikawa S, Haraguchi N, Sakai D, Satoh T, Doki Y, Mori M. Cancer stem cell theory in gastrointestinal malignancies: recent progress and upcoming challenges. J Gastroenterol. 2011;46(10):1145-57.

27. Yoshida GJ, Saya H. Therapeutic strategies targeting cancer stem cells. Cancer Sci. 2016;107(1):5-11.

28. Scheel C, Weinberg RA. Cancer stem cells and epithelial-mesenchymal transition: concepts and molecular links. Semin Cancer Biol. 2012;22(5-6): 396-403.

29. Yoshida GJ, Saya H. Inversed relationship between CD44 variant and c-Myc due to oxidative stress-induced canonical Wnt activation. Biochem Biophys Res Commun. 2014;443(2):622-7.

30. Demitrack ES, Gifford GB, Keeley TM, Carulli AJ, VanDussen KL, Thomas D, Giordano TJ, Liu Z, Kopan R, Samuelson LC. Notch signaling regulates gastric antral LGR5 stem cell function. EMBO J. 2015;34(20):2522-36.

31. Singh SS, Yap WN, Arfuso F, Kar S, Wang C, Cai W, Dharmarajan AM, Sethi G, Kumar AP. Targeting the PIJK/Akt signaling pathway in gastric carcinoma: a reality for personalized medicine? World I Gastroenterol. 2015;21 (43):12261-73.

32. Tang X, Zheng D, Hu P, Zeng Z, Li M, Tucker L, Monahan R, Resnick MB, Liu M, Ramratnam B. Glycogen synthase kinase 3 beta inhibits microRNA-18396-182 cluster via the beta-Catenin/TCF/LEF-1 pathway in gastric cancer cells. Nucleic Acids Res. 2014;42(5):2988-98.

33. Chikano Y, Domoto T, Furuta T, Sabit H, Kitano-Tamura A, Pyko IV, Takino T, Sai Y, Hayashi Y, Sato H, et al. Glycogen synthase kinase 3beta sustains invasion of glioblastoma via the focal adhesion kinase, Rac1, and c-Jun Nterminal kinase-mediated pathway. Mol Cancer Ther. 2015;14(2):564-74.

\section{Submit your next manuscript to BioMed Central and we will help you at every step:}

- We accept pre-submission inquiries

- Our selector tool helps you to find the most relevant journal

- We provide round the clock customer support

- Convenient online submission

- Thorough peer review

- Inclusion in PubMed and all major indexing services

- Maximum visibility for your research

Submit your manuscript at www.biomedcentral.com/submit

) Biomed Central 